\title{
Transient global amnesia. Have you considered hyperhomocysteinemia?
}

\author{
Amnésia global transitória. Foi considerada hiper-homocisteinemia?
}

\author{
Elias E. MAZOKOPAKIS ${ }^{1}$
}

\section{Dear Editor,}

I read with great interest the article by Alessandro et al. ${ }^{1}$ about the predictors of recurrence among patients having previously experienced transient global amnesia (TGA). With this letter, I would like to present the characteristics (medical history, clinical and laboratory findings) of five patients (three women, two men) diagnosed with TGA according to all known Hodges and Warlow criteria in a Greek hospital during the last five years. All patients were young, nonsmokers, with negative personal medical history for cardiovascular disease, seizures, brain tumor or migraine. Two of the three women reported two consecutive spontaneous early pregnancy losses and one possible previous TGA event. The memory loss during TGA episodes was sudden, unrelated to previous intense psychological stress, and its duration was between 12 and 24 hours (Table). Neurological examination of the patients was normal; a mild polyneuropathy was diagnosed in the two men. Brain magnetic resonance imaging, including diffusion weighted imaging sequences, electroencephalography, electrocardiography and echocardiography, were also normal. An ultrasound examination of both sides of the carotid arteries showed only a mild arteriosclerosis, but no plaques or severe stenosis. Laboratory investigations revealed normal values of hemoglobin and hematocrit, normal renal, liver and thyroid functions, normal serum total cholesterol and low-density lipoprotein levels, low serum folate $(<4 \mathrm{ng} / \mathrm{mL})$ and vitamin B12 $(<300 \mathrm{pg} / \mathrm{mL})$ levels, and high serum total homocysteine (tHcy) levels (>15 $\mu \mathrm{mol} / \mathrm{L}$ ) (Table). All the patients were diagnosed with mutations of methylenetetrahydrofolate reductase (MTHFR) gene (hereditary hyperhomocysteinemia) (Table) and received initially L-methylfolate ( 1 gr per os daily) and hydroxocobalamin acetate (1 gr IM; daily for seven days, weekly for four weeks, then monthly). After four months of treatment, serum folate and vitamin B12 levels were increased, but serum tHcy levels had decreased significantly (Table). However, a lifelong intake of L-methylfolate supplement to control the MTHFR mutation gene-induced hyperhomocysteinemia was suggested to all patients. Although a causal relationship between TGA episodes and

Table.Characteristics of the five patients with TGA.

\begin{tabular}{|c|c|c|c|c|c|}
\hline Variables & Patient 1 & Patient 2 & Patient 3 & Patient 4 & Patient 5 \\
\hline Age (years)/ Sex & $51 /$ female & $43 /$ female & 34 / female & $48 /$ male & $57 /$ male \\
\hline Duration of memory loss (hours) & 12 & 17 & 22 & 24 & 19 \\
\hline \multicolumn{6}{|l|}{ Folate (normal value: > $4 \mathrm{ng} / \mathrm{mL}$ ) } \\
\hline Before treatment $(\mathrm{ng} / \mathrm{mL})$ & 1.4 & 2.8 & 3.1 & 0.5 & 2.9 \\
\hline After treatment (ng/mL) & 8.2 & 10.8 & 11.4 & 7.1 & 9.3 \\
\hline \multicolumn{6}{|c|}{ Vitamin B12 (normal value: > 300 pg/mL) } \\
\hline Before treatment $(\mathrm{pg} / \mathrm{mL})$ & 123 & 156 & 218 & 122 & 234 \\
\hline After treatment (pg/mL) & 987 & 1100 & 1270 & 930 & 1380 \\
\hline \multicolumn{6}{|l|}{ tHcy (normal value: $<15 \mu \mathrm{mol} / \mathrm{L}$ ) } \\
\hline Before treatment $(\mu \mathrm{mol} / \mathrm{L})$ & 30.4 & 26.3 & 22.5 & 34.2 & 26.5 \\
\hline After treatment ( $\mu \mathrm{mol} / \mathrm{L})$ & 12.4 & 10.3 & 9.7 & 13.8 & 11.9 \\
\hline \multicolumn{6}{|l|}{ MTHFR gene mutations } \\
\hline C677T & Homozygous & Heterozygous & Homozygous & Homozygous & Homozygous \\
\hline A1298C & - & Heterozygous & - & Heterozygous & - \\
\hline
\end{tabular}

Hcy: total homocysteine; MTHFR: methylenetetrahydrofolate reductase.

${ }^{1}$ Naval Hospital of Crete, Department of Internal Medicine, Chania, Greece.

Elias E. Mazokopakis (iD) https://orcid.org/0000-0003-3698-4884

Correspondence: Elias E. Mazokopakis; Naval Hospital of Crete, Souda, Chania, 73 200, Crete, Greece; E-mail: emazokopakis@yahoo.gr

Conflict of interest: There is no conflict of interest to declare.

Received 28 March 2019; Received in final form 12 June 2019; Accepted 06 August 2019. 
hyperhomocysteinemia cannot be proven (small sample), some cases, like ours, have been reported ${ }^{2-4}$, probably due to hyperhomocysteinemia-induced hippocampal dysfunction or vascular disorders. Moreover, it is known that hyperhomocysteinemia is a strong modifiable risk factor for vascular dementia and Alzheimer's disease ${ }^{5}$. However, it is unclear whether elevated levels of tHcy or the related vitamin deficiency, either alone or in combination, is responsible for cognitive decline ${ }^{6}$. I believe that serum folate, vitamin B12 and tHcy levels should be controlled in all patients with TGA and managed appropriately. Further multicenter clinical studies are required to confirm our findings.

\section{References}

1. Alessandro L, Calandri IL, Suarez MF, Heredia ML, Chaves H, Allegri RF, et al. Transient global amnesia: clinical features and prognostic factors suggesting recurrence. Arq Neuropsiquiatr. 2019 Jan;77(1):3-9. https://doi.org/10.1590/0004-282×20180157

2. Semmler A, Klein A, Moskau S, Linnebank M. Transient global amnesia-like episode in a patient with severe hyperhomocysteinemia. Eur J Neurol. 2007 Sep;14(9):e5-6. https://doi.org/10.1111/j.1468-1331.2007.01707.x

3. Khan R, Hossain MA, Nai Q, Yousif AM, Sen S. Hyperhomocysteinemia association with transient global amnesia: A rare case report. N Am J Med Sci. 2015 Aug;7(8):374-6. https://doi.org/10.4103/1947-2714.163647
4. Portaro S, Naro A, Cimino V, Maresca G,

Corallo F, Morabito R, et al. Risk factors of transient global amnesia: three case reports. Medicine (Baltimore). 2018 Oct;97(41):e12723. https://doi.org/10.1097/MD.0000000000012723

5. Smith AD, Refsum H. Homocysteine, B vitamins, and cognitive impairment. Annu Rev Nutr. 2016 Jul;36(1):211-39. https://doi.org/10.1146/annurev-nutr-071715-050947

6. Ansari R, Mahta A, Mallack E, Luo JJ. Hyperhomocysteinemia and neurologic disorders: a review.J Clin Neurol. 2014 Oct;10(4):281-8. https://doi.org/10.3988/jcn.2014.10.4.281 